Supporting Information

\title{
Neuroprotective Effects of Curcumin in Cerebral Ischemia: Cellular and Molecular Mechanisms
}

\section{Lalita Subedi and Bhakta Prasad Gaire*}

Department of Anesthesiology and Neurology, Shock Trauma and Anesthesiology Research Center, University of Maryland, School of Medicine, Baltimore, Maryland, 21201, USA.

Corresponding author: Bhakta Prasad Gaire, PhD, samarpanbp@gmail.com 
Table S1: Neuroprotective effects of curcumin in cerebral ischemia with associated mechanism of actions.

\begin{tabular}{|c|c|c|c|c|c|c|}
\hline S.N. & Intervention & $\begin{array}{l}\text { Dose, route, } \\
\text { species }\end{array}$ & $\begin{array}{l}\text { Ischemia } \\
\text { models }\end{array}$ & Experimental evidences & $\begin{array}{l}\text { Involved } \\
\text { mechanisms }\end{array}$ & Reference(s) \\
\hline 1 & $\begin{array}{l}\text { PNIPAM } \\
\text { (curcumin } \\
\text { nanoparticle) }\end{array}$ & $\begin{array}{l}100 \mu \mathrm{g} / \mathrm{kg} \text {, } \\
\text { intranasal, rats }\end{array}$ & $\mathrm{MCAO}$ & $\begin{array}{l}\uparrow \text { Spontaneous behavioral } \\
\text { activities, } \downarrow \text { infarct volume and } \\
\text { neurodegeneration, } \downarrow \text { lipid } \\
\text { peroxidation }\end{array}$ & Antioxidant & 1 \\
\hline 2 & $\begin{array}{l}\text { CNB-001 } \\
\text { pyrazole } \\
\text { derivative }\end{array}$ & $\begin{array}{l}10 \mathrm{mg} / \mathrm{kg}, \mathrm{IV} \text {, } \\
\text { rabbits }\end{array}$ & $\begin{array}{l}\text { Spinal } \\
\text { cord } \\
\text { ischemia }\end{array}$ & $\downarrow$ Paraplegia & NA & 2 \\
\hline 3 & Curcumin & $\begin{array}{l}100 \mathrm{mg} / \mathrm{kg}, \mathrm{PO}, \\
\text { rats }\end{array}$ & MCAO & $\begin{array}{l}\downarrow \text { Neurological deficits and brain } \\
\text { infarction, } \downarrow \text { lipid peroxidation, } \\
\text { calcium levels, superoxide } \\
\text { dismutase }\end{array}$ & Antioxidant & 3 \\
\hline 4 & $\begin{array}{l}\text { CNB-001 } \\
\text { pyrazole } \\
\text { derivative }\end{array}$ & $\begin{array}{l}10 \mathrm{mg} / \mathrm{kg}, \mathrm{IV}, \\
\text { rabbits, monkeys }\end{array}$ & $\begin{array}{l}\text { embolized } \\
\text { models }\end{array}$ & $\begin{array}{l}\downarrow \text { Behavior deficits and stroke } \\
\text { expansion, } \downarrow \text { COC } 2 \text {, VCAM, 5- } \\
\text { LOX, } \uparrow \text { pERK and BDNF }\end{array}$ & NA & 4 \\
\hline 5 & $\begin{array}{l}\text { Curcumin }+ \\
\text { vagus } \\
\text { nerve } \\
\text { stimulation }\end{array}$ & $\begin{array}{l}50 \mathrm{mg} / \mathrm{kg}, \mathrm{IP}, \\
\text { rats }\end{array}$ & MCAOR & $\begin{array}{l}\uparrow \text { Learning and memory, } \downarrow \\
\text { neurological deficits, and infarct } \\
\text { volume, } \downarrow \text { apoptosis, } \downarrow \\
\text { inflammatory cytokines, and } \uparrow \\
\text { survival proteins }\end{array}$ & $\begin{array}{l}\text { Anti-apoptotic, } \\
\text { Anti- } \\
\text { inflammatory }\end{array}$ & 5 \\
\hline 6 & Curcumin & $\begin{array}{l}20,100 \mu \mathrm{M}, \\
\mathrm{BMEC}\end{array}$ & OGD & $\begin{array}{l}\uparrow \text { Trans-endothelial electric } \\
\text { resistance, } \uparrow \text { ZO- } 1 \text { occludin, and } \\
\text { HO- } 1 \text { expression }\end{array}$ & $\begin{array}{l}\text { BBB protectant, } \\
\text { Antioxidant }\end{array}$ & 6 \\
\hline 7 & Curcumin & $\begin{array}{l}100,200 \mathrm{mg} / \mathrm{kg}, \\
\mathrm{PO}, \text { rats and } \\
\text { PC } 12 \text { cells }\end{array}$ & $\begin{array}{l}\text { MCAO } \\
\text { (rats) and } \\
\text { OGD } \\
\text { (PC12 } \\
\text { cells) }\end{array}$ & $\begin{array}{l}\downarrow \text { Neurological deficits and } \\
\text { infarct volume, } \downarrow \text { autophagy } \\
\text { proteins LC3 and p62, } \downarrow \text { caspase- } \\
3, \downarrow \text { serum LDH after MCAO, } \downarrow \\
\text { apoptotic cell number and } \\
\text { caspase- } 3 \text { in PC } 12 \text { cells }\end{array}$ & $\begin{array}{l}\text { Anti-apoptotic, } \\
\text { Anti-autophagy }\end{array}$ & 7 \\
\hline 8 & Curcumin & $\begin{array}{l}10 \mu \mathrm{M}, \mathrm{IP}, \text { rats, } \\
\text { hippocampal } \\
\text { neuronal culture } \\
\text { or } \mathrm{SH}-\mathrm{SY} 5 \mathrm{Y} \\
\text { cells, } 50 \mathrm{mg} / \mathrm{kg} \text {, } \\
\mathrm{IP} \text {, rats }\end{array}$ & $\begin{array}{l}\text { OGD/ } \\
\text { glutamate } \\
\text { toxicity, } \\
\text { MCAO }\end{array}$ & $\begin{array}{l}\downarrow \text { IL-1 } \beta, \downarrow \text { ROS, } \downarrow \text { glutamate } \\
\text { toxicity, reducing pPERK, } \\
\text { pIRE1 } \alpha, \uparrow \text { pAMPK, } \downarrow \text { TXNIP, } \\
\text { NLRP3 inflammasome, cleaved } \\
\text { caspase-1, IL-6, } \uparrow \text { mitochondrial } \\
\text { membrane potential, } \downarrow \text { caspase-3, } \\
\downarrow \text { apoptotic cells, } \downarrow \text { brain } \\
\text { infarction and neurological } \\
\text { deficits, } \downarrow \text { pIRE1 } \alpha, \text { pPERK, } \\
\text { NLRP3 after MCAO }\end{array}$ & $\begin{array}{l}\text { Anti- } \\
\text { inflammatory, } \\
\text { Antioxidant, } \\
\text { Anti-apoptotic }\end{array}$ & 8 \\
\hline 9 & Curcumin & $20 \mu \mathrm{M}, \mathrm{BMECs}$ & OGD & $\begin{array}{l}\downarrow \text { OGD-induced cell injury, } \downarrow \text { IL- } \\
1 \beta, \downarrow \text { LDH release, } \downarrow \text { pp38, } \\
\text { pJNK, NF- } \kappa B\end{array}$ & $\begin{array}{l}\text { Anti- } \\
\text { inflammatory }\end{array}$ & 9 \\
\hline 10 & Curcumin & $\begin{array}{l}50 \mathrm{mg} / \mathrm{kg}, \mathrm{IP}, \\
\text { rats }\end{array}$ & MCAO & $\uparrow$ Gamma enolase & Antioxidant & 10 \\
\hline
\end{tabular}




\begin{tabular}{|c|c|c|c|c|c|c|}
\hline 11 & Curcumin & $\begin{array}{l}150,300 \mathrm{mg} / \mathrm{kg} \text {, } \\
\mathrm{IP} \text {, mice }\end{array}$ & SAH & $\begin{array}{l}\downarrow \text { Brain injury and vascular } \\
\text { inflammation, } \downarrow \text { NF- } \kappa \text { B, } \downarrow \text { COX- } \\
2, \text { IL- } 1 \beta \text {, IL- } 6 \text {, TNF- } \alpha \text {, iNOS, } \\
\text { VCAM, ICAM- } 1, \downarrow \text { superoxide, } \\
\text { nitrotyrosine formation or lipid } \\
\text { peroxidation }\end{array}$ & $\begin{array}{l}\text { Anti- } \\
\text { inflammatory, } \\
\text { Antioxidant }\end{array}$ & 11 \\
\hline 12 & Curcumin & $\begin{array}{l}300 \mathrm{mg} / \mathrm{kg}, \mathrm{IP} \text {, } \\
\text { rats }\end{array}$ & MCAO & 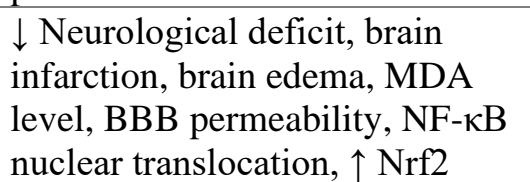 & $\begin{array}{l}\text { Anti- } \\
\text { inflammatory, } \\
\text { Antioxidant }\end{array}$ & 12 \\
\hline 13 & Curcumin & $\begin{array}{l}50,100 \mathrm{mg} / \mathrm{kg}, \\
\text { PO, rats }\end{array}$ & BCCAO & $\begin{array}{l}\downarrow \text { Neurological deficits, motor } \\
\text { deficits, restores memory object } \\
\text { recognition, short-term and long- } \\
\text { term memory, } \uparrow \text { hippocampal } \\
\text { neuronal density }\end{array}$ & NA & 13 \\
\hline 14 & Curcumin & $\begin{array}{l}1-15 \mu \mathrm{M}, \mathrm{SH}- \\
\text { SY5Y cells }\end{array}$ & OGD/R & $\begin{array}{l}\downarrow \text { Cytotoxicity, } \downarrow \text { cell death, } \\
\text { caspase-3 activity, Bax } 3 \text { and } \uparrow \\
\text { Bc12 expression, } \uparrow \text { APE1/Ref-1 } \\
\text { expression, } \downarrow \text { NOX } 2, \uparrow \\
\text { PI3K/AKT }\end{array}$ & $\begin{array}{l}\text { Anti-apoptotic, } \\
\text { Antioxidant }\end{array}$ & 14 \\
\hline 15 & Curcumin & $\begin{array}{l}40 \mathrm{mg} / \mathrm{kg}, \\
\text { gavage, rats }\end{array}$ & $\begin{array}{l}\text { diabetic rat } \\
\text { MCAO }\end{array}$ & $\begin{array}{l}\downarrow \text { Brain infarction, } \\
\text { neurodegeneration, neurological } \\
\text { deficits, brain edema, apoptotic } \\
\text { cell death, Bax expression, } \uparrow \\
\text { GLUT1 and GLUT3 } \\
\text { translocation and Bcl2 } \\
\text { expression }\end{array}$ & Anti-apoptotic & 15 \\
\hline 16 & Curcumin & $\begin{array}{l}100,300,500 \\
\mathrm{mg} / \mathrm{kg}, \mathrm{IP}, \text { rats }\end{array}$ & MCAO & $\begin{array}{l}\downarrow \text { Infarct volume, ox-LDL and } \\
\text { MDA levels, } \uparrow \text { Bcl-2, } \\
\text { mitochondrial cytochrome c, } \downarrow \\
\text { cytosolic cytochrome c and } \\
\text { cleaved caspase- } 3\end{array}$ & $\begin{array}{l}\text { Antioxidant, } \\
\text { Anti-apoptotic }\end{array}$ & 16 \\
\hline 17 & Curcumin & $\begin{array}{l}50,100 \mathrm{mg} / \mathrm{kg} \text {, } \\
\text { IP, rats }\end{array}$ & $2 \mathrm{VO}$ & $\begin{array}{l}\uparrow \text { Memory and cognition } \\
\text { function, } \uparrow \text { hippocampal } \\
\text { neuronal survival, } \uparrow \text { serum HDL } \\
\text { and total cholesterol }\end{array}$ & Antioxidant & 17 \\
\hline 18 & Curcumin & $\begin{array}{l}150 \mathrm{mg} / \mathrm{kg}, \mathrm{IP}, \\
\text { rats }\end{array}$ & MCAO & $\begin{array}{l}\downarrow \text { GADD } 153 \text { and caspase- } 12 \\
\text { positive cells and their protein } \\
\text { expression, }\end{array}$ & ER-stress & 18 \\
\hline 19 & Curcumin & $\begin{array}{l}10,50 \mathrm{mg} / \mathrm{kg}, \mathrm{IP}, \\
\text { rats }\end{array}$ & pMCAO & $\begin{array}{l}\downarrow \text { Brain infarction, neurological } \\
\text { deficit, neurodegeneration, brain } \\
\text { water content, MPO levels, } \\
\text { TLR } 2 \text { and TLR } 4 \text { expression, NF- } \\
\kappa B \text { expression, TNF- } \alpha \text { and IL-1 } \\
\text { production }\end{array}$ & $\begin{array}{l}\text { Anti- } \\
\text { inflammatory }\end{array}$ & 19 \\
\hline 20 & $\begin{array}{l}\text { Curcumin and } \\
\text { nanoparticles }\end{array}$ & $\begin{array}{l}25,50 \mathrm{mg} / \mathrm{kg} \\
\mathrm{PO}, \text { rats }\end{array}$ & BCCAO & $\begin{array}{l}\downarrow \text { Neurological deficits, and } \\
\text { acetylcholinesterase levels, } \uparrow \\
\text { mitochondrial complex activity, } \\
\downarrow \text { LPO, nitrite levels and increase } \\
\text { GSH, SOD, and catalase activity }\end{array}$ & Antioxidant & 20 \\
\hline
\end{tabular}




\begin{tabular}{|c|c|c|c|c|c|c|}
\hline 21 & Curcumin & $\begin{array}{l}50 \mathrm{mg} / \mathrm{kg}, \mathrm{IP}, \\
\text { rats }\end{array}$ & MCAOR & $\begin{array}{l}\downarrow \text { Neurological deficits, brain } \\
\text { edema and brain infarction, } \uparrow \\
\text { Sirt1 and Bcl } 2 \text { expression, } \downarrow \text { p53 } \\
\text { and Bax expression, } \downarrow \text { TNF- } \alpha \\
\text { and IL- } 6 \text { production, } \uparrow \\
\text { mitochondrial cytochrome c and } \\
\downarrow \text { cytosolic cytochrome c }\end{array}$ & $\begin{array}{l}\text { Anti-apoptotic, } \\
\text { Anti- } \\
\text { inflammatory }\end{array}$ & 21 \\
\hline 22 & Curcumin & $\begin{array}{l}50,100 \mathrm{mg} / \mathrm{kg} \text {, } \\
\text { IP, rats }\end{array}$ & MCAOR & $\begin{array}{l}\downarrow \text { Neurological deficits and brain } \\
\text { infarction, } \uparrow \text { neuronal density, } \uparrow \\
\text { mitochondrial viability, } \uparrow \text { UCP } 2 \\
\text { expression, } \uparrow \text { NRF- } 1 \text { and TFAM } \\
1 \text { expression }\end{array}$ & $\begin{array}{l}\text { Mitochondrial } \\
\text { stability }\end{array}$ & 22 \\
\hline 23 & Curcumin & $\begin{array}{l}300 \mathrm{mg} / \mathrm{kg}, \mathrm{IP}, \\
\text { rats }\end{array}$ & MCAOR & $\begin{array}{l}\downarrow \text { Brain infarction, ICAM-1, } \\
\text { MMP-9, caspase- } 3 \text { and NF- } \kappa \text { B } \\
\text { expression }\end{array}$ & $\begin{array}{l}\text { BBB protectant } \\
\text { Anti- } \\
\text { inflammatory }\end{array}$ & 23 \\
\hline 24 & Curcumin & $\begin{array}{l}80 \mathrm{mg} / \mathrm{kg}, \mathrm{IP}, \\
\text { rats }\end{array}$ & MCAOR & $\begin{array}{l}\downarrow \text { Neurological deficits, brain } \\
\text { infarction, neurodegeneration, } \\
\text { brain water content, IL-1 } \beta \text {, IL-8, } \\
\text { pJAK2, and pSTAT3 expression }\end{array}$ & $\begin{array}{l}\text { Anti- } \\
\text { inflammatory }\end{array}$ & 24 \\
\hline 25 & Curcumin & $\begin{array}{l}100 \mathrm{mg} / \mathrm{kg}, \mathrm{IP}, \\
\text { rats }\end{array}$ & $4 \mathrm{VO}$ & $\downarrow$ Retinal cell apoptosis & Anti-apoptotic & 25 \\
\hline 26 & Curcumin & $\begin{array}{l}150 \mathrm{mg} / \mathrm{kg}, \mathrm{IP}, \\
\text { mice }\end{array}$ & dMCAO & $\begin{array}{l}\downarrow \text { Brain infarction, motor } \\
\text { function deficit, } \downarrow \text { TNF- } \alpha \text {, IL-12, } \\
\text { CD16, CD } 32 \text {, iNOS expression, } \\
\uparrow C D 206, \text { Arg- } 1, \text { Ym } 1 / 2 \\
\text { expression, } \downarrow \text { M1 polarization, } \uparrow \\
\text { M2 polarization }\end{array}$ & $\begin{array}{l}\text { Anti- } \\
\text { inflammatory }\end{array}$ & 26 \\
\hline 27 & Curcumin & $\begin{array}{l}0.25-1 \mu \mathrm{M}, \\
\text { primary neuronal } \\
\text { culture }\end{array}$ & OGD & $\begin{array}{l}\downarrow \text { LDH release, caspase- } 3 \\
\text { activity, } \uparrow \text { flotillin activity, } \uparrow \\
\text { pERK, } \downarrow \text { pJNK, }\end{array}$ & Anti-apoptotic & 27 \\
\hline \multirow[t]{2}{*}{28} & Curcumin & $\begin{array}{l}100-400 \mathrm{mg} / \mathrm{kg} \text {, } \\
\mathrm{IP}, \text { mice }\end{array}$ & MCAOR & $\begin{array}{l}\downarrow \text { Brain infarction and } \\
\text { neurological deficit, } \uparrow \text { neuronal } \\
\text { cell density, } \downarrow \text { neuronal } \\
\text { apoptosis, } \uparrow \text { Bcl- } 2, \downarrow \text { Bax and } \\
\text { caspase- } 3 \text { expression }\end{array}$ & Anti-apoptotic & 28 \\
\hline & & $\begin{array}{l}5-35 \mu \mathrm{M}, \mathrm{N} 2 \mathrm{a} \\
\text { cells }\end{array}$ & OGD/R & $\begin{array}{l}\uparrow \text { Cell viability, } \downarrow \text { apoptosis, } \uparrow \\
\text { Bcl- } 2, \downarrow \text { Bax, Bad, and caspase- } 3 \\
\text { activity }\end{array}$ & Anti-apoptotic & 28 \\
\hline 29 & Curcumin & $\begin{array}{l}200 \mathrm{mg} / \mathrm{kg}, \mathrm{IP} \text {, } \\
\text { rats }\end{array}$ & MCAOR & $\begin{array}{l}\downarrow \text { PPARy expression, infarct } \\
\text { volume and neurological deficit, } \\
\uparrow \text { neuronal density, } \downarrow \text { IL- } 1 \beta, \\
\text { TNF- } \alpha \text {, PGE2 and NO } \\
\text { production, } \downarrow \text { COX-2 and iNOS } \\
\text { expression, } \downarrow \text { NF- } \kappa \text { B signaling }\end{array}$ & $\begin{array}{l}\text { Anti- } \\
\text { inflammatory }\end{array}$ & 29 \\
\hline 30 & Curcumin & $\begin{array}{l}100 \mathrm{mg} / \mathrm{kg}, \mathrm{IP} \\
\text { mice }\end{array}$ & $\mathrm{ICH}$ & $\begin{array}{l}\downarrow \text { Neurological deficit and brain } \\
\text { water content, } \downarrow \text { T lymphocytes } \\
\text { infiltration, } \downarrow \text { VCAM expression, } \\
\downarrow \text { IFN } \gamma \text { and IL- } 17 \text { expression }\end{array}$ & $\begin{array}{l}\text { Anti- } \\
\text { inflammatory, } \\
\text { BBB protectant }\end{array}$ & 30 \\
\hline 31 & Curcumin & $\begin{array}{l}25,50 \mathrm{mg} / \mathrm{kg} \\
\mathrm{PO}, \text { rats }\end{array}$ & left CCAO & $\begin{array}{l}\downarrow \text { CA } 1 \text { and CA } 3 \text { neuronal death, } \\
\downarrow \text { COX- } 2, \text { TNF- } \alpha \text { expression }\end{array}$ & $\begin{array}{l}\text { Anti- } \\
\text { inflammatory }\end{array}$ & 31 \\
\hline
\end{tabular}




\begin{tabular}{|c|c|c|c|c|c|c|}
\hline 32 & Curcumin & $\begin{array}{l}0.5-8 \mu \mathrm{M}, \\
\text { primary neurons }\end{array}$ & $\begin{array}{l}\text { Hypoxia } \\
\text { reperfusion }\end{array}$ & $\begin{array}{l}\uparrow \text { Cell viability, } \downarrow \text { apoptosis, } \downarrow \\
\text { IL- } 6 \text {, TNF- } \alpha \text {, Wnt5a, pJNK, p65 } \\
\text { and caspase- } 3 \text { expression }\end{array}$ & $\begin{array}{l}\text { Anti-apoptotic, } \\
\text { anti- } \\
\text { inflammatory }\end{array}$ & 32 \\
\hline 33 & Curcumin & $\begin{array}{l}50 \mathrm{mg} / \mathrm{kg}, \mathrm{IP}, \\
\text { rats }\end{array}$ & pMCAO & $\begin{array}{l}\uparrow \text { Protein phosphatase } 2 \mathrm{~A} \\
\text { expression }\end{array}$ & NA & 33 \\
\hline 34 & Curcumin & $\begin{array}{l}50,100 \mathrm{mg} / \mathrm{kg}, \\
\text { IP, rats }\end{array}$ & pMCAO & $\begin{array}{l}\downarrow \text { Neurological deficits, brain } \\
\text { infarction, and brain water } \\
\text { content, } \uparrow \text { Nrf- } 2 \text { and HO-1 } \\
\text { expression, }\end{array}$ & Antioxidant & 34 \\
\hline 35 & Curcumin & $\begin{array}{l}10-200 \mu \mathrm{g} / \mathrm{g}, \mathrm{IP} \\
\text { mice }\end{array}$ & left CCAO & $\begin{array}{l}\downarrow \text { Brain infarction, apoptotic } \\
\text { neuronal death, } \uparrow \text { astroglial } \\
\text { activation, } \downarrow \text { microglial } \\
\text { activation, } \downarrow \text { pSTAT3 signaling, } \\
\uparrow \text { prohibitin expression }\end{array}$ & Anti-apoptotic & 35 \\
\hline 36 & Curcumin & $\begin{array}{l}1-50 \mu \mathrm{M}, \mathrm{PC} 12 \\
\text { cells }\end{array}$ & $\begin{array}{l}\text { Glutamate } \\
\text { toxicity }\end{array}$ & $\begin{array}{l}\downarrow \text { Glutathione peroxidase } 1 \text {, } \\
\text { glutathione disulfide, } \mathrm{Ca}^{2+} \\
\text { influx, nitric oxide production, } \\
\text { cytochrome c release, Bax/Bcl-2 } \\
\text { ratio, caspase- } 3 \text { activity, lactate } \\
\text { dehydrogenase release, reactive } \\
\text { oxygen species, } \mathrm{H}_{2} \mathrm{O}_{2} \text {, and } \\
\text { malondialdehyde, } \downarrow \text { glutathione, } \\
\text { glutathione reductase, SOD and } \\
\text { catalase }\end{array}$ & $\begin{array}{l}\text { Anti-glutamate } \\
\text { toxicity } \\
\text { Antioxidant }\end{array}$ & 36 \\
\hline 37 & Curcuminoids & $\begin{array}{l}300 \mathrm{mg} / \mathrm{kg}, \mathrm{IP}, \\
\text { rats }\end{array}$ & MCAOR & $\begin{array}{l}\downarrow \text { Bran infarction, cerebral } \\
\text { edema and neurological deficits, } \\
\downarrow \text { neutrophil adhesion to } \\
\text { endothelial cells and rolling } \downarrow \\
\text { ICAM- } 1 \text { and NF- } \mathrm{B}, \uparrow \\
\text { microcirculation }\end{array}$ & $\begin{array}{l}\text { Anti- } \\
\text { inflammatory, } \\
\text { BBB protectant }\end{array}$ & 37 \\
\hline \multirow[t]{2}{*}{38} & $\begin{array}{l}\text { CNB-001 } \\
\text { pyrazole } \\
\text { derivatives }\end{array}$ & $\begin{array}{l}100 \mathrm{mg} / \mathrm{kg}, \mathrm{SC}, \\
\text { rabbits }\end{array}$ & embolism & $\begin{array}{l}\uparrow \text { Motor functions, } \uparrow \text { pAKT, } \\
\text { pERK, ORP150 and CaMKIIa }\end{array}$ & NA & 38 \\
\hline & & $1-10 \mu \mathrm{M}$ & $\begin{array}{l}\text { HT-22 } \\
\text { neuron }\end{array}$ & $\begin{array}{l}\downarrow \text { IAA- and glutamate-induced } \\
\text { cytotoxicity, } \uparrow \text { pAKT and pERK } \\
\text { expression }\end{array}$ & NA & 38 \\
\hline 39 & Curcumin & $\begin{array}{l}100-300 \mathrm{mg} / \mathrm{kg}, \\
\mathrm{IP}, \text { rats }\end{array}$ & $\begin{array}{l}\text { thrombus } \\
\text { MCAO }\end{array}$ & $\begin{array}{l}\downarrow \text { neurological deficits, brain } \\
\text { infarction, } \uparrow \text { GSH-px, } \downarrow \text { MPO } \\
\text { activity, DCFH-DA, DHR123, } \\
\text { DAF2-DA }\end{array}$ & Antioxidant & 39 \\
\hline 40 & $\begin{array}{l}\text { Curcumin and } \\
\text { Candesartan }\end{array}$ & $\begin{array}{l}\text { cur } 60 \mu \mathrm{g} / \mathrm{kg}+ \\
\text { candestran } 50 \\
\mu \mathrm{g} / \mathrm{kg}, \mathrm{IP}, \text { mice }\end{array}$ & MCAOR & $\begin{array}{l}\text { Restore SOD and GST level, } \uparrow \\
\text { blood flow, reduce brain } \\
\text { infarction }\end{array}$ & Antioxidant & 40 \\
\hline 41 & Curcumin & $\begin{array}{l}1-50 \mu \mathrm{g} / \mathrm{ml} \\
\text { PC12 Cells }\end{array}$ & $\begin{array}{l}\text { Glucose } \\
\text { serum } \\
\text { deprivation }\end{array}$ & $\uparrow$ Cell viability & NA & 41 \\
\hline 42 & Curcumin & $\begin{array}{l}40 \mathrm{mg} / \mathrm{kg}, \mathrm{IP}, \\
\text { rats }\end{array}$ & HI model & $\begin{array}{l}\downarrow \text { Brain edema, } \downarrow \text { NOS activity } \\
\text { and AQP-4 expression }\end{array}$ & NA & 42 \\
\hline
\end{tabular}




\begin{tabular}{|c|c|c|c|c|c|c|}
\hline 43 & $\begin{array}{l}\text { Hexahydro } \\
\text { curcumin }\end{array}$ & $\begin{array}{l}40 \mathrm{mg} / \mathrm{kg}, \mathrm{IP}, \\
\text { rats }\end{array}$ & MCAOR & $\begin{array}{l}\downarrow \text { Neurological deficits, brain } \\
\text { infarction, } \uparrow \text { claudin and } \\
\text { occludin expression, } \downarrow \text { BBB } \\
\text { permeability, } \downarrow \text { ICAM- } 1 \text {, } \\
\text { VCAM- } 1 \text { expression and MPO } \\
\text { activity }\end{array}$ & $\begin{array}{l}\text { Anti- } \\
\text { inflammatory, } \\
\text { BBB protectant, } \\
\text { Antioxidant }\end{array}$ & 43 \\
\hline 44 & $\begin{array}{l}\text { Hexahydro } \\
\text { curcumin }\end{array}$ & $\begin{array}{l}10-40 \mathrm{mg} / \mathrm{kg}, \mathrm{IP}, \\
\text { rats }\end{array}$ & MCAOR & $\begin{array}{l}\downarrow \text { Neurological deficits and brain } \\
\text { infarction, } \downarrow \text { neurodegeneration, } \\
\downarrow \text { MDA levels, p65 and COX-2 } \\
\text { expression, } \downarrow \text { NO release, } \uparrow \text { Nrf- } \\
2, \text { HO- } 1 \text { expression, SOD, GSH, } \\
\text { and GSH-px production }\end{array}$ & $\begin{array}{l}\text { Antioxidant, } \\
\text { Anti- } \\
\text { inflammatory }\end{array}$ & 44 \\
\hline 45 & Curcumin & $\begin{array}{l}200 \mathrm{mg} / \mathrm{kg}, \mathrm{IP}, \\
\text { rats }\end{array}$ & BCCAO & $\begin{array}{l}\uparrow \mathrm{CA} 1 \text { neuronal density, } \uparrow \mathrm{GSH} \\
\text { and } \downarrow \text { MDA levels, } \uparrow \text { SOD and } \\
\text { catalase production, }\end{array}$ & Antioxidant & 45 \\
\hline \multirow[t]{2}{*}{46} & $\begin{array}{l}\text { Curcumin } \\
\text { nanoparticles }\end{array}$ & $\begin{array}{l}25 \mathrm{mg} / \mathrm{kg}, \mathrm{IV} \\
\text { mice }\end{array}$ & MCAOR & $\begin{array}{l}\downarrow \text { MMP9, } \uparrow \text { occludin and ZO- } 1 \text {, } \\
\downarrow \text { M1 microglia, TNF- } \alpha \text { and IL- } \\
1 \beta \text { secretion, } \downarrow \text { caspase-3 } \\
\text { expression, } \downarrow \text { brain infarction } \\
\text { and neurological deficits }\end{array}$ & $\begin{array}{l}\text { BBB protectant, } \\
\text { Anti-apoptotic, } \\
\text { Anti- } \\
\text { inflammatory }\end{array}$ & 46 \\
\hline & & $20 \mu \mathrm{M}$ & MBMECs & $\begin{array}{l}\uparrow \text { Transendothelial electrical } \\
\text { resistance }\end{array}$ & BBB protectant & 46 \\
\hline 47 & $\begin{array}{l}\text { Curcumin } \\
\text { nanoparticles }\end{array}$ & $\begin{array}{l}5 \mathrm{mg} / \mathrm{kg}(200 \mu \mathrm{g} \\
\text { of curcumin }), \\
\text { PO, rats }\end{array}$ & BCCAO & $\begin{array}{l}\uparrow \text { Hippocampal neuronal density, } \\
\downarrow \text { ROS production, lipid } \\
\text { peroxidation and NADH } \\
\text { activity, } \uparrow \text { SDH, SOD and CAT } \\
\text { activity, } \downarrow \text { TUNEL positive cells, } \\
\downarrow \text { mitochondrial Bax, and } \uparrow \\
\text { mitochondrial Cytochrome C, } \uparrow \\
\text { Bcl-2, } \downarrow \text { caspase-9, caspase-3, } \\
\text { COX- } 2 \text { and iNOS expression }\end{array}$ & $\begin{array}{l}\text { Antioxidant, } \\
\text { Anti-apoptotic, } \\
\text { Anti- } \\
\text { inflammatory }\end{array}$ & 47 \\
\hline 48 & Curcumin & $\begin{array}{l}150 \mathrm{mg} / \mathrm{kg}, \mathrm{IP} \\
\text { mice }\end{array}$ & $\mathrm{ICH}$ & $\begin{array}{l}\downarrow \text { Evans blue extravasation, } \downarrow \\
\text { water content, } \downarrow \\
\text { neurodegeneration, } \downarrow \text { ventricle } \\
\text { size, } \downarrow \text { neurological dysfunction, } \\
\downarrow \text { BBB dysfunction, and } \downarrow \text { MMP } \\
\text { activity }\end{array}$ & BBB protectant & 48 \\
\hline \multirow[t]{2}{*}{49} & Curcumin & $\begin{array}{l}300 \mathrm{mg} / \mathrm{kg}, \mathrm{IP}, \\
\text { rats }\end{array}$ & MCAOR & $\begin{array}{l}\downarrow \text { Infarct volume, } \downarrow \text { MDA, } \uparrow \\
\text { SOD } \uparrow \text { Nrf- } 2, \uparrow \text { pAKT, NQO1 }\end{array}$ & Antioxidant & 49 \\
\hline & & $\begin{array}{l}2.5-50 \mu \mathrm{M}, \\
\text { Primary cortical } \\
\text { neurons }\end{array}$ & OGD/R & $\begin{array}{l}\uparrow \text { Cell viability, } \downarrow \text { LDH level, } \uparrow \\
\text { NQO1 expression }\end{array}$ & Antioxidant & 49 \\
\hline 50 & Curcumin & $\begin{array}{l}200 \mathrm{mg} / \mathrm{kg}, \mathrm{IP} \\
\text { rats }\end{array}$ & MCAOR & $\begin{array}{l}\downarrow \text { Neurological deficits, motor } \\
\text { deficits, } \uparrow \text { MAP2 density, } \downarrow \text { brain } \\
\text { infarction, } \downarrow \text { LC3 positive } \\
\text { neurons, } \uparrow \text { pAKT, pMTOR, } \downarrow \\
\text { LC3II/I, } \downarrow \text { TLR } 4, \text { pp } 38, \text { IL-1, IL- } \\
6, \text { TNF- } \alpha, \text { iNOS expression }\end{array}$ & $\begin{array}{l}\text { Anti-autophagy, } \\
\text { Anti- } \\
\text { inflammatory }\end{array}$ & 50 \\
\hline 51 & Curcumin & $\begin{array}{l}300 \mathrm{mg} / \mathrm{kg}, \mathrm{IP}, \\
\text { rats }\end{array}$ & MCAOR & $\begin{array}{l}\downarrow \text { Brain infarction, brain edema, } \\
\downarrow \text { MDA and GPx level, restore }\end{array}$ & Antioxidant & 51 \\
\hline
\end{tabular}




\begin{tabular}{|c|c|c|c|c|c|c|}
\hline & & & & $\begin{array}{l}\text { glutathione peroxidase, } \uparrow \text { SOD } \\
\text { activity } \downarrow \text { peroxynitrite }\end{array}$ & & \\
\hline 52 & Curcumin & $\begin{array}{l}1,2 \mathrm{mg} / \mathrm{kg}, \mathrm{IV}, \\
\text { rats }\end{array}$ & MCAOR & $\begin{array}{l}\downarrow \text { Brain infarction, neurological } \\
\text { deficits, } \uparrow \text { survival, } \downarrow \text { brain water } \\
\text { content, } \downarrow \text { iNOS, } \downarrow \text { NO and } \downarrow \\
\text { LDH release }\end{array}$ & $\begin{array}{l}\text { Anti- } \\
\text { inflammatory }\end{array}$ & 52 \\
\hline 53 & Curcumin & $\begin{array}{l}300 \mathrm{mg} / \mathrm{kg}, \mathrm{IP}, \\
\text { rats }\end{array}$ & MCAOR & $\begin{array}{l}\downarrow \text { Brain infarction, degenerating } \\
\text { neurons, TUNEL positive cells, } \\
\text { caspase-positive cells, and } \\
\text { caspase-3 expression }\end{array}$ & Anti-apoptotic & 53 \\
\hline \multirow[t]{2}{*}{54} & Curcumin & $\begin{array}{l}100,300 \mathrm{mg} / \mathrm{kg} \\
\mathrm{IP} \text {, rats }\end{array}$ & MCAOR & $\begin{array}{l}\downarrow \text { Neurological deficits, brain } \\
\text { water content, brain infarction, } \\
\text { hippocampal neuronal } \\
\text { degeneration, TUNEL cells, } \uparrow \\
\text { pMEK, pERK, pCREB, } \uparrow \text { Bcl } 2 \\
\text { and } \downarrow \text { Bax expression }\end{array}$ & Anti-apoptotic & 54 \\
\hline & & $\begin{array}{l}5-20 \mu \mathrm{M} \\
\text { Primary } \\
\text { astrocytes }\end{array}$ & OGD/R & $\begin{array}{l}\uparrow \text { Cell viability, } \downarrow \text { LDH release, } \uparrow \\
\text { pMEK, pERK, pCREB, } \uparrow \text { Bcl } 2 \\
\text { and } \downarrow \text { Bax expression }\end{array}$ & Anti-apoptotic & 54 \\
\hline 55 & Curcumin & $\begin{array}{l}5 \mu \mathrm{M}, \mathrm{UN}-\mathrm{SH}- \\
\text { SY5Y, RA-SH- } \\
\text { SY5Y cells }\end{array}$ & $\begin{array}{l}\mathrm{H}_{2} \mathrm{O}_{2}, 6- \\
\text { OHDA } \\
\text { toxicity }\end{array}$ & $\begin{array}{l}\uparrow \text { Cell viability against } \mathrm{H}_{2} \mathrm{O}_{2} \\
\text { toxicity, } \uparrow \text { cell viability against } \\
\text { 6-OHDA toxicity }\end{array}$ & Antioxidant & 55 \\
\hline 56 & Curcumin & $\begin{array}{l}30 \mathrm{mg} / \mathrm{kg}, \mathrm{IP} \\
\text { gerbils }\end{array}$ & BCCAO & $\begin{array}{l}\uparrow \text { CA1 neuronal density, } \downarrow \text { GFAP } \\
\text { and Isolectin-B positive cells, } \downarrow \\
\text { HNE and Cytochrome C positive } \\
\text { cells in hippocampus, } \uparrow \\
\text { mitochondrial membrane } \\
\text { potential } \downarrow \text { cleaved caspase-3 } \\
\text { expression, decrease TUNEL } \\
\text { positive cells, } \uparrow \text { locomotor } \\
\text { activity }\end{array}$ & Anti-apoptotic & 56 \\
\hline 57 & $\begin{array}{l}\text { PNIPAM } \\
\text { (curcumin } \\
\text { nanoparticle) }\end{array}$ & $\begin{array}{l}100 \mu \mathrm{g} / \mathrm{kg}, \\
\text { IV/IN/ Wistar } \\
\text { rats }\end{array}$ & MCAOR & $\begin{array}{l}\downarrow \text { Neurological deficits, } \downarrow \text { TNF- } \alpha \\
\text { and IL- } 1 \beta \text { production }\end{array}$ & $\begin{array}{l}\text { Anti- } \\
\text { inflammatory }\end{array}$ & 57 \\
\hline 58 & Curcumin & $\begin{array}{l}25 \mathrm{mg} / \mathrm{kg}, \mathrm{IP} \\
\text { Albino rats }\end{array}$ & MCAOR & $\begin{array}{l}\uparrow \text { Bcl-2, Sirt } 1, \downarrow \text { p53 and Bax } \\
\text { expression, } \downarrow \text { TNF- } \alpha \text { and IL-6 } \\
\text { production, } \downarrow \text { brain water content } \\
\text { and ROS amount, } \uparrow \\
\text { mitochondrial membrane } \\
\text { potential } \downarrow \text { brain infarction and } \\
\text { neurological deficits }\end{array}$ & $\begin{array}{l}\text { Anti-apoptotic, } \\
\text { Anti- } \\
\text { inflammatory }\end{array}$ & 58 \\
\hline 59 & Curcumin & $\begin{array}{l}300 \mathrm{mg} / \mathrm{kg}, \mathrm{IP} \text {, } \\
\text { rats }\end{array}$ & MCAOR & $\begin{array}{l}\uparrow \text { Prdx6 expression, } \downarrow \\
\text { neurological deficits and brain } \\
\text { infarction, } \downarrow \text { ROS, MDA, } \\
\text { nitrotyrosine, and } 8-\mathrm{OHdG}\end{array}$ & Antioxidant & 59 \\
\hline 60 & Curcumin & $\begin{array}{l}50,100,200 \\
\mathrm{mg} / \mathrm{kg} \text { IP, rats }\end{array}$ & BCCAO & $\begin{array}{l}\downarrow \text { Xanthine oxidase and } \\
\text { superoxide activity, } \downarrow \mathrm{O}_{2} \text { free } \\
\text { radical, and MDA, } \uparrow \mathrm{GSH} \text { level }\end{array}$ & Antioxidant & 60 \\
\hline 61 & Curcumin & $\begin{array}{l}100,500 \mathrm{ng} / \mathrm{ml}, \\
\text { HT22 neurons }\end{array}$ & OGD/R & $\begin{array}{l}\downarrow \text { Apoptosis, intracellular ROS } \\
\text { and mitochondrial superoxide }\end{array}$ & $\begin{array}{l}\text { Anti-apoptotic } \\
\text { Antioxidant }\end{array}$ & 61 \\
\hline
\end{tabular}


accumulation, and $\downarrow$ intracellular

SOD2, MMP, and mitochondrial

complex I activity

\begin{tabular}{|c|c|c|c|c|c|c|}
\hline 62 & Curcumin & $\begin{array}{l}40 \mathrm{mg} / \mathrm{kg}, \mathrm{IV}, \\
\text { rats }\end{array}$ & $\begin{array}{l}\text { MCAO } \\
\text { embolus } \\
\text { injection }\end{array}$ & $\begin{array}{l}\downarrow \text { TNF- } \alpha, \text { IL-1 } \beta, \text { IL-6 } \\
\text { production, HMGB1, pJAK2, } \\
\text { pSTAT3 }\end{array}$ & $\begin{array}{l}\text { Anti- } \\
\text { inflammatory }\end{array}$ & 62 \\
\hline 63 & $\begin{array}{l}\text { Tetrahydroxy } \\
\text { curcumin }\end{array}$ & $\begin{array}{l}2,10,25 \mathrm{mg} / \mathrm{kg} \text {, } \\
\mathrm{IP}, \text { mice }\end{array}$ & $4-\mathrm{VO}$ & $\begin{array}{l}\downarrow \text { Neurological deficits, } \uparrow \text { CA1 } \\
\text { neuronal viability, } \uparrow \text { SOD level, } \\
\downarrow \text { MDA }\end{array}$ & Antioxidant & 63 \\
\hline 64 & $\begin{array}{l}\text { Curcumin } \\
\text { loaded } \\
\text { exosomes }\end{array}$ & $\begin{array}{l}50 \mathrm{ug} / \mathrm{mice}, \mathrm{IV}, \\
\text { mice }\end{array}$ & MCAOR & $\begin{array}{l}\downarrow \text { TNF- } \alpha, \text { IL-1 } \beta, \text { IL- } 6 \text {, expression } \\
\text { and production, } \downarrow \text { Iba } 1^{+} \text {cells, } \\
\downarrow \text { pp } 65 \text { and cleaved caspase-3 } \\
\text { expression }\end{array}$ & $\begin{array}{l}\text { Anti- } \\
\text { inflammatory }\end{array}$ & 64 \\
\hline 65 & $\begin{array}{l}\text { Tetrahydro } \\
\text { curcumin }\end{array}$ & $\begin{array}{l}25 \mathrm{mg} / \mathrm{kg}, \mathrm{IP}, \\
\text { mice }\end{array}$ & MCAOR & $\begin{array}{l}\downarrow \text { Brain infarction, neurological } \\
\text { deficits, brain edema, } \downarrow \text { Evans } \\
\text { blue leakage, } \downarrow \text { SAHH and } \uparrow \\
\text { MTHFR, } \downarrow \text { Hcy, p47phox, } \\
\text { MMP-9, LC3II/I, SAHH, and } \\
\text { MTHFR, } \downarrow \text { MMP }^{+} \text {cells }\end{array}$ & $\begin{array}{l}\text { Antioxidant, } \\
\text { Anti-autophagy } \\
\text { BBB protectant }\end{array}$ & 65 \\
\hline 66 & $\begin{array}{l}\text { Tetrahydro } \\
\text { curcumin }\end{array}$ & $\begin{array}{l}25 \mathrm{mg} / \mathrm{kg}, \mathrm{IP}, \\
\text { mice }\end{array}$ & MCAOR & $\begin{array}{l}\downarrow \text { Neurological deficits, } \downarrow \text { Evans } \\
\text { blue leakage, fluorescent protein } \\
\text { leakage, } \uparrow \text { vascular area, } \downarrow \text { Hcy, } \\
\uparrow \text { MTHFR and } \downarrow \text { SAHH, } \downarrow \text { p47, } \\
\text { gp91, Trx- } 2 \text {, and } \uparrow \text { SOD activity, } \\
\uparrow \text { mitochondrial ATP and O2 } \\
\text { consumption, } \downarrow \text { mitochondrial } \\
\text { permeability }\end{array}$ & $\begin{array}{l}\text { Antioxidant, } \\
\text { BBB protectant, } \\
\text { Mitochondrial } \\
\text { dysfunction }\end{array}$ & 66 \\
\hline 67 & Curcumin & $\begin{array}{l}1.25-20 \mu \mathrm{M} \\
\text { PC12 cells }\end{array}$ & OGD/R & $\begin{array}{l}\uparrow \text { Cell viability and } \downarrow \text { apoptosis } \\
\text { rate, } \downarrow \text { LC3II/I and HIF-1 } \alpha, \uparrow \\
\text { p62 }\end{array}$ & $\begin{array}{l}\text { Anti-autophagy } \\
\text { and anti-apoptotic }\end{array}$ & 67 \\
\hline
\end{tabular}

\section{References}

1. Ahmad, N.; Umar, S.; Ashafaq, M.; Akhtar, M.; Iqbal, Z.; Samim, M.; Ahmad, F. J., A comparative study of PNIPAM nanoparticles of curcumin, demethoxycurcumin, and bisdemethoxycurcumin and their effects on oxidative stress markers in experimental stroke. Protoplasma 2013, 250 (6), 1327-38.

2. Lapchak, P. A.; Boitano, P. D.; Bombien, R.; Chou, D.; Knight, M.; Muehle, A.; Winkel, M. T.; Khoynezhad, A., CNB-001 reduces paraplegia in rabbits following spinal cord ischemia. Neural Regen Res 2019, 14 (12), 2192-2198.

3. Shukla, P. K.; Khanna, V. K.; Ali, M. M.; Khan, M. Y.; Srimal, R. C., Anti-ischemic effect of curcumin in rat brain. Neurochem Res 2008, 33 (6), 1036-43.

4. Lapchak, P. A.; Boitano, P. D.; Bombien, R.; Cook, D. J.; Doyan, S.; Lara, J. M.; Schubert, D. R., CNB-001, a pleiotropic drug is efficacious in embolized agyrencephalic New Zealand white rabbits and ischemic gyrencephalic cynomolgus monkeys. Exp Neurol 2019, 313, 98-108.

5. Xu, J.; Kong, X.; Xiu, H.; Dou, Y.; Wu, Z.; Sun, P., Combination of curcumin and vagus nerve stimulation attenuates cerebral ischemia/reperfusion injury-induced behavioral deficits. Biomed Pharmacother 2018, 103, 614-620. 
6. Wang, Y. F.; Gu, Y. T.; Qin, G. H.; Zhong, L.; Meng, Y. N., Curcumin ameliorates the permeability of the blood-brain barrier during hypoxia by upregulating heme oxygenase-1 expression in brain microvascular endothelial cells. J Mol Neurosci 2013, 51 (2), 344-51.

7. Zhang, Y.; Fang, M.; Sun, Y.; Zhang, T.; Shi, N.; Li, J.; Jin, L.; Liu, K.; Fu, J., Curcumin attenuates cerebral ischemia injury in Sprague-Dawley rats and PC12 cells by suppressing overactivated autophagy. $J$ Photochem Photobiol B 2018, 184, 1-6.

8. Li, Y.; Li, J.; Li, S.; Li, Y.; Wang, X.; Liu, B.; Fu, Q.; Ma, S., Curcumin attenuates glutamate neurotoxicity in the hippocampus by suppression of ER stress-associated TXNIP/NLRP3 inflammasome activation in a manner dependent on AMPK. Toxicol Appl Pharmacol 2015, 286 (1), 53-63.

9. Dong, H. J.; Shang, C. Z.; Peng, D. W.; Xu, J.; Xu, P. X.; Zhan, L.; Wang, P., Curcumin attenuates ischemia-like injury induced IL-1beta elevation in brain microvascular endothelial cells via inhibiting MAPK pathways and nuclear factor-kappaB activation. Neurol Sci 2014, 35 (9), 1387-92.

10. Gim, S. A.; Lee, S. R.; Shah, F. A.; Koh, P. O., Curcumin attenuates the middle cerebral artery occlusion-induced reduction in gamma-enolase expression in an animal model. Lab Anim Res 2015, 31 (4), 198-203.

11. Wakade, C.; King, M. D.; Laird, M. D.; Alleyne, C. H., Jr.; Dhandapani, K. M., Curcumin attenuates vascular inflammation and cerebral vasospasm after subarachnoid hemorrhage in mice. Antioxid Redox Signal 2009, 11 (1), 35-45.

12. Li, W.; Suwanwela, N. C.; Patumraj, S., Curcumin by down-regulating NF-kB and elevating Nrf2, reduces brain edema and neurological dysfunction after cerebral I/R. Microvasc Res 2016, 106, 117-27.

13. Kamali Dolatabadi, L.; Emamghoreishi, M.; Namavar, M. R.; Badeli Sarkala, H., Curcumin Effects on Memory Impairment and Restoration of Irregular Neuronal Distribution in the Hippocampal CA1 Region After Global Cerebral Ischemia in Male Rats. Basic Clin Neurosci 2019, 10 (5), 527-539.

14. Wu, L.; Jiang, C.; Kang, Y.; Dai, Y.; Fang, W.; Huang, P., Curcumin exerts protective effects against hypoxiareoxygenation injury via the enhancement of apurinic/apyrimidinic endonuclease 1 in SHSY5Y cells: Involvement of the PI3K/AKT pathway. Int J Mol Med 2020, 45 (4), 993-1004.

15. Xia, M.; Ye, Z.; Shi, Y.; Zhou, L.; Hua, Y., Curcumin improves diabetes mellitusassociated cerebral infarction by increasing the expression of GLUT1 and GLUT3. Mol Med Rep 2018, 17 (1), 1963-1969.

16. Zhao, J.; Yu, S.; Zheng, W.; Feng, G.; Luo, G.; Wang, L.; Zhao, Y., Curcumin improves outcomes and attenuates focal cerebral ischemic injury via antiapoptotic mechanisms in rats. Neurochem Res 2010, 35 (3), 374-9.

17. Tian, M.; Zhang, X.; Wang, L.; Li, Y., Curcumin induces ABCA1 expression and apolipoprotein A-Imediated cholesterol transmembrane in the chronic cerebral hypoperfusion aging rats. Am J Chin Med 2013, 41 (5), 1027-42.

18. Zhu, H.; Fan, Y.; Sun, H.; Chen, L.; Man, X., Curcumin inhibits endoplasmic reticulum stress induced by cerebral ischemia-reperfusion injury in rats. Exp Ther Med 2017, 14 (5), 4047-4052.

19. Tu, X. K.; Yang, W. Z.; Chen, J. P.; Chen, Y.; Ouyang, L. Q.; Xu, Y. C.; Shi, S. S., Curcumin inhibits TLR2/4-NF-kappaB signaling pathway and attenuates brain damage in permanent focal cerebral ischemia in rats. Inflammation 2014, 37 (5), 1544-51.

20. Kakkar, V.; Muppu, S. K.; Chopra, K.; Kaur, I. P., Curcumin loaded solid lipid nanoparticles: an efficient formulation approach for cerebral ischemic reperfusion injury in rats. Eur J Pharm Biopharm 2013, 85 (3 Pt A), 339-45.

21. Miao, Y.; Zhao, S.; Gao, Y.; Wang, R.; Wu, Q.; Wu, H.; Luo, T., Curcumin pretreatment attenuates inflammation and mitochondrial dysfunction in experimental stroke: The possible role of Sirt1 signaling. Brain Res Bull 2016, 121, 9-15.

22. Liu, L.; Zhang, W.; Wang, L.; Li, Y.; Tan, B.; Lu, X.; Deng, Y.; Zhang, Y.; Guo, X.; Mu, J.; Yu, G., Curcumin prevents cerebral ischemia reperfusion injury via increase of mitochondrial biogenesis. Neurochem Res 2014, 39 (7), 1322-31. 
23. Li, W.; Suwanwela, N. C.; Patumraj, S., Curcumin prevents reperfusion injury following ischemic stroke in rats via inhibition of NFkappaB, ICAM-1, MMP-9 and caspase-3 expression. Mol Med Rep 2017, $16(4), 4710-4720$.

24. Li, L.; Li, H.; Li, M., Curcumin protects against cerebral ischemia-reperfusion injury by activating JAK2/STAT3 signaling pathway in rats. Int J Clin Exp Med 2015, 8 (9), 14985-91.

25. Wang, S.; Ye, Q.; Tu, J.; Zhang, M.; Ji, B., Curcumin protects against hypertension aggravated retinal ischemia/reperfusion in a rat stroke model. Clin Exp Hypertens 2017, 39 (8), 711-717.

26. Liu, Z.; Ran, Y.; Huang, S.; Wen, S.; Zhang, W.; Liu, X.; Ji, Z.; Geng, X.; Ji, X.; Du, H.; Leak, R. K.; $\mathrm{Hu}, \mathrm{X}$., Curcumin Protects against Ischemic Stroke by Titrating Microglia/Macrophage Polarization. Front Aging Neurosci 2017, 9, 233.

27. Lu, Z.; Liu, Y.; Shi, Y.; Shi, X.; Wang, X.; Xu, C.; Zhao, H.; Dong, Q., Curcumin protects cortical neurons against oxygen and glucose deprivation/reoxygenation injury through flotillin-1 and extracellular signal-regulated kinase1/2 pathway. Biochem Biophys Res Commun 2018, 496 (2), 515-522.

28. Xie, C. J.; Gu, A. P.; Cai, J.; Wu, Y.; Chen, R. C., Curcumin protects neural cells against ischemic injury in N2a cells and mouse brain with ischemic stroke. Brain Behav 2018, 8 (2), e00921.

29. Liu, Z. J.; Liu, W.; Liu, L.; Xiao, C.; Wang, Y.; Jiao, J. S., Curcumin Protects Neuron against Cerebral Ischemia-Induced Inflammation through Improving PPAR-Gamma Function. Evid Based Complement Alternat Med 2013, 2013, 470975.

30. Liu, W.; Yuan, J.; Zhu, H.; Zhang, X.; Li, L.; Liao, X.; Wen, Z.; Chen, Y.; Feng, H.; Lin, J., Curcumin reduces brain-infiltrating $T$ lymphocytes after intracerebral hemorrhage in mice. Neurosci Lett 2016, 620, 74-82.

31. de Alcantara, G. F.; Simoes-Neto, E.; da Cruz, G. M.; Nobre, M. E.; Neves, K. R.; de Andrade, G. M.; Brito, G. A.; Viana, G. S., Curcumin reverses neurochemical, histological and immuno-histochemical alterations in the model of global brain ischemia. J Tradit Complement Med 2017, 7 (1), 14-23.

32. Zhou, J.; Wu, N.; Lin, L., Curcumin Suppresses Apoptosis and Inflammation in Hypoxia/Reperfusion-Exposed Neurons via Wnt Signaling Pathway. Med Sci Monit 2020, 26, e920445.

33. Shah, F. A.; Park, D. J.; Gim, S. A.; Koh, P. O., Curcumin treatment recovery the decrease of protein phosphatase 2A subunit B induced by focal cerebral ischemia in Sprague-Dawley rats. Lab Anim Res 2015, $31(3), 134-8$.

34. Yang, C.; Zhang, X.; Fan, H.; Liu, Y., Curcumin upregulates transcription factor Nrf2, HO-1 expression and protects rat brains against focal ischemia. Brain Res 2009, 1282, 133-41.

35. Rocha-Ferreira, E.; Sisa, C.; Bright, S.; Fautz, T.; Harris, M.; Contreras Riquelme, I.; Agwu, C.; Kurulday, T.; Mistry, B.; Hill, D.; Lange, S.; Hristova, M., Curcumin: Novel Treatment in Neonatal HypoxicIschemic Brain Injury. Front Physiol 2019, 10, 1351.

36. Chang, C. H.; Chen, H. X.; Yu, G.; Peng, C. C.; Peng, R. Y., Curcumin-Protected PC12 Cells Against Glutamate-Induced Oxidative Toxicity. Food Technol Biotechnol 2014, 52 (4), 468-478.

37. Funk, J. L.; Frye, J. B.; Davis-Gorman, G.; Spera, A. L.; Bernas, M. J.; Witte, M. H.; Weinand, M. E.; Timmermann, B. N.; McDonagh, P. F.; Ritter, L., Curcuminoids limit neutrophil-mediated reperfusion injury in experimental stroke by targeting the endothelium. Microcirculation 2013, 20 (6), 544-54.

38. Lapchak, P. A.; Schubert, D. R.; Maher, P. A., Delayed treatment with a novel neurotrophic compound reduces behavioral deficits in rabbit ischemic stroke. J Neurochem 2011, 116 (1), 122-31.

39. Dohare, P.; Garg, P.; Jain, V.; Nath, C.; Ray, M., Dose dependence and therapeutic window for the neuroprotective effects of curcumin in thromboembolic model of rat. Behav Brain Res 2008, 193 (2), 289-97.

40. Awad, A. S., Effect of combined treatment with curcumin and candesartan on ischemic brain damage in mice. J Stroke Cerebrovasc Dis 2011, 20 (6), 541-8. 
41. Alinejad, B.; Ghorbani, A.; Sadeghnia, H. R., Effects of combinations of curcumin, linalool, rutin, safranal, and thymoquinone on glucose/serum deprivation-induced cell death. Avicenna J Phytomed 2013, 3 (4), 321-8.

42. Yu, L.; Yi, J.; Ye, G.; Zheng, Y.; Song, Z.; Yang, Y.; Song, Y.; Wang, Z.; Bao, Q., Effects of curcumin on levels of nitric oxide synthase and AQP-4 in a rat model of hypoxia-ischemic brain damage. Brain Res 2012, 1475, 88-95.

43. Wicha, P.; Tocharus, J.; Janyou, A.; Jittiwat, J.; Chaichompoo, W.; Suksamrarn, A.; Tocharus, C., Hexahydrocurcumin alleviated blood-brain barrier dysfunction in cerebral ischemia/reperfusion rats. Pharmacol Rep 2020, 72 (3), 659-671.

44. Wicha, P.; Tocharus, J.; Janyou, A.; Jittiwat, J.; Changtam, C.; Suksamrarn, A.; Tocharus, C., Hexahydrocurcumin protects against cerebral ischemia/reperfusion injury, attenuates inflammation, and improves antioxidant defenses in a rat stroke model. PLoS One 2017, 12 (12), e0189211.

45. Al-Omar, F. A.; Nagi, M. N.; Abdulgadir, M. M.; Al Joni, K. S.; Al-Majed, A. A., Immediate and delayed treatments with curcumin prevents forebrain ischemia-induced neuronal damage and oxidative insult in the rat hippocampus. Neurochem Res 2006, 31 (5), 611-8.

46. Wang, Y.; Luo, J.; Li, S. Y., Nano-Curcumin Simultaneously Protects the Blood-Brain Barrier and Reduces M1 Microglial Activation During Cerebral Ischemia-Reperfusion Injury. ACS Appl Mater Interfaces 2019, 11 (4), 3763-3770.

47. Mukherjee, A.; Sarkar, S.; Jana, S.; Swarnakar, S.; Das, N., Neuro-protective role of nanocapsulated curcumin against cerebral ischemia-reperfusion induced oxidative injury. Brain Res 2019, $1704,164-173$.

48. Sun, Y.; Dai, M.; Wang, Y.; Wang, W.; Sun, Q.; Yang, G. Y.; Bian, L., Neuroprotection and sensorimotor functional improvement by curcumin after intracerebral hemorrhage in mice. J Neurotrauma 2011, 28 (12), 2513-21.

49. Wu, J.; Li, Q.; Wang, X.; Yu, S.; Li, L.; Wu, X.; Chen, Y.; Zhao, J.; Zhao, Y., Neuroprotection by curcumin in ischemic brain injury involves the Akt/Nrf2 pathway. PLoS One 2013, 8 (3), e59843.

50. Huang, L.; Chen, C.; Zhang, X.; Li, X.; Chen, Z.; Yang, C.; Liang, X.; Zhu, G.; Xu, Z., Neuroprotective Effect of Curcumin Against Cerebral Ischemia-Reperfusion Via Mediating Autophagy and Inflammation. $J$ Mol Neurosci 2018, 64 (1), 129-139.

51. Thiyagarajan, M.; Sharma, S. S., Neuroprotective effect of curcumin in middle cerebral artery occlusion induced focal cerebral ischemia in rats. Life Sci 2004, 74 (8), 969-85.

52. Jiang, J.; Wang, W.; Sun, Y. J.; Hu, M.; Li, F.; Zhu, D. Y., Neuroprotective effect of curcumin on focal cerebral ischemic rats by preventing blood-brain barrier damage. Eur J Pharmacol 2007, 561 (1-3), 54-62.

53. Zhao, J.; Zhao, Y.; Zheng, W.; Lu, Y.; Feng, G.; Yu, S., Neuroprotective effect of curcumin on transient focal cerebral ischemia in rats. Brain Res 2008, 1229, 224-32.

54. Xu, L.; Ding, L.; Su, Y.; Shao, R.; Liu, J.; Huang, Y., Neuroprotective effects of curcumin against rats with focal cerebral ischemia-reperfusion injury. Int J Mol Med 2019, 43 (4), 1879-1887.

55. Jantas, D.; Chwastek, J.; Grygier, B.; Lason, W., Neuroprotective Effects of Necrostatin-1 Against Oxidative Stress-Induced Cell Damage: an Involvement of Cathepsin D Inhibition. Neurotox Res 2020, 37 (3), 525-542.

56. Wang, Q.; Sun, A. Y.; Simonyi, A.; Jensen, M. D.; Shelat, P. B.; Rottinghaus, G. E.; MacDonald, R. S.; Miller, D. K.; Lubahn, D. E.; Weisman, G. A.; Sun, G. Y., Neuroprotective mechanisms of curcumin against cerebral ischemia-induced neuronal apoptosis and behavioral deficits. J Neurosci Res 2005, 82 (1), 138-48.

57. Ahmad, N.; Ahmad, I.; Umar, S.; Iqbal, Z.; Samim, M.; Ahmad, F. J., PNIPAM nanoparticles for targeted and enhanced nose-to-brain delivery of curcuminoids: UPLC/ESI-Q-ToF-MS/MS-based 
pharmacokinetics and pharmacodynamic evaluation in cerebral ischemia model. Drug Deliv 2016, 23 (7), 2095-2114.

58. Zhang, Y.; Yan, Y.; Cao, Y.; Yang, Y.; Zhao, Q.; Jing, R.; Hu, J.; Bao, J., Potential therapeutic and protective effect of curcumin against stroke in the male albino stroke-induced model rats. Life Sci 2017, $183,45-49$.

59. Jia, G.; Tan, B.; Ma, J.; Zhang, L.; Jin, X.; Li, C., Prdx6 Upregulation by Curcumin Attenuates Ischemic Oxidative Damage via SP1 in Rats after Stroke. Biomed Res Int 2017, 2017, 6597401.

60. Ghoneim, A. I.; Abdel-Naim, A. B.; Khalifa, A. E.; El-Denshary, E. S., Protective effects of curcumin against ischaemia/reperfusion insult in rat forebrain. Pharmacol Res 2002, 46 (3), 273-9.

61. Wang, Y.; Zhang, Y.; Yang, L.; Yuan, J.; Jia, J.; Yang, S., SOD2 Mediates Curcumin-Induced Protection against Oxygen-Glucose Deprivation/Reoxygenation Injury in HT22 Cells. Evid Based Complement Alternat Med 2019, 2019, 2160642.

62. Wu, Y.; Xu, J.; Xu, J.; Zheng, W.; Chen, Q.; Jiao, D., Study on the mechanism of JAK2/STAT3 signaling pathway-mediated inflammatory reaction after cerebral ischemia. Mol Med Rep 2018, 17 (4), 5007-5012.

63. Lin, B.; Yu, H.; Lin, Y.; Cai, C.; Lu, H.; Zhu, X., Suppression of GRASP65 phosphorylation by tetrahydrocurcumin protects against cerebral ischemia/reperfusion injury via ERK signaling. Mol Med Rep 2016, 14 (5), 4775-4780.

64. Tian, T.; Zhang, H. X.; He, C. P.; Fan, S.; Zhu, Y. L.; Qi, C.; Huang, N. P.; Xiao, Z. D.; Lu, Z. H.; Tannous, B. A.; Gao, J., Surface functionalized exosomes as targeted drug delivery vehicles for cerebral ischemia therapy. Biomaterials 2018, 150, 137-149.

65. Tyagi, N.; Qipshidze, N.; Munjal, C.; Vacek, J. C.; Metreveli, N.; Givvimani, S.; Tyagi, S. C., Tetrahydrocurcumin ameliorates homocysteinylated cytochrome-c mediated autophagy in hyperhomocysteinemia mice after cerebral ischemia. J Mol Neurosci 2012, 47 (1), 128-38.

66. Mondal, N. K.; Behera, J.; Kelly, K. E.; George, A. K.; Tyagi, P. K.; Tyagi, N., Tetrahydrocurcumin epigenetically mitigates mitochondrial dysfunction in brain vasculature during ischemic stroke. Neurochem Int 2019, 122, 120-138.

67. Hou, Y.; Wang, J.; Feng, J., The neuroprotective effects of curcumin are associated with the regulation of the reciprocal function between autophagy and HIF-1alpha in cerebral ischemia-reperfusion injury. Drug Des Devel Ther 2019, 13, 1135-1144. 